\title{
Deutsche Sicherheitsinteressen im 21. Jahrhundert
}

\author{
Egon Bahr*
}

\begin{abstract}
Within the last 20 years historical changes have strong influence on global events. Environmental threats, changes in the global power structure, the fight for resources, the American dissociation from the United Nations and terrorism are challenges to the world community.

These problems, in addition to continuation of the European process of integration and the positioning of Germany within the EU, pose difficulties for the German foreign and security policy.

Germany can protect its security interests by a return to a »healthy patriotism « and a deliberate and self-confident relationship with the concept of power. This could enable Germany to make an important contribution to global security.
\end{abstract}

Keywords: Sicherheitspolitik, Deutschland, nationale Interessen

$\mathrm{W}$ er 1906 versucht hätte, die Rolle des Deutschen Reiches für das 20. Jahrhundert zu beschreiben, wäre kläglich gescheitert. Das Ende des Deutschen Reiches und des Zarentums lagen hinter dem Horizont, erst recht eine Sowjetunion, auf die sich die Aufmerksamkeit der Welt fast für das ganze Jahrhundert richten sollte. Der Begriff Kalter Krieg existierte noch nicht. Das Ende des Kolonialismus war kaum vorstellbar. Rundfunk und Fernsehen, die Spaltung des Atoms, verwendbar für Energienutzung wie für Waffen, Interkontinentalraketen, Satelliten im nahen Weltraum, die Landung auf dem Mond und das Handy konnten nicht bekannt sein.

Nichts spricht dafür, dass die Explosion des Wissens zu Ende ist. Die Geschwindigkeit dürfte eher zunehmen, mit der die Menschheit versuchen wird, die Summe ihres Nichtwissens zu verkleinern, besonders auf den Feldern der Physik, Chemie und Biologie. Wenig spricht dafür, dass die Struktur des Menschen sich ändert, alles zu machen, was er kann. Auch der alte Adam hat sich globalisiert. Ein neuer Mensch ist dabei nicht herausgekommen. Alles spricht für gefährliche Verknotungen und Unberechenbarkeiten. Eine solide Prognose für das Jahrhundert ist unmöglich.

Wer also vorsichtiger ist und sich auf erkennbare Entwicklungen für die nächsten 20 Jahre beschränkt, wird auch dies nur mit dem Hinweis können, dass in den letzten 20 Jahren, also seit 1986, überaus viel geschehen ist, einschließlich welthistorischer Umbrüche. Die Wahrscheinlichkeit, dass die Geschichte eine Pause einlegt, ist gering. Der globale Befund mit dem Blick auf die nächsten 20 Jahre lässt sich in vier Kategorien einteilen.

1. Die Umweltgefährdung wird zunehmen. Die Klimaerwärmung ist messbar geworden. Die Knappheit an Wasser wird fühlbarer werden. Ob das Wachstum der Weltbevölkerung zu stoppen ist, bleibt nicht prognostizierbar; das gilt auch für Spannungen zwischen den älter werdenden reichen Gesellschaften und armen mit ihrem Geburtenreichtum. Der Hun-

\footnotetext{
* Prof. Egon Bahr, Bundesminister a.D.
}

ger wird nicht besiegt werden. Alle diese Probleme - und die Aufzählung erhebt keinen Anspruch auf Vollständigkeit - sind global.

Vernunft und Logik drängen, sie durch Zusammenarbeit zu regeln, sie mindestens durch globale Konventionen und regionale Vereinbarungen beherrschbar zu machen. Auch wenn die Erfahrung warnt, dass diese Probleme militärisch nicht »lösbar« sind, bleiben Kriege nicht auszuschließen. Der Kern der europäischen Idee, Macht dem Recht unterzuordnen, heißt Zusammenarbeit. Das globale Gewicht Europas kann wachsen, sofern Zusammenarbeit das Schlüsselwort des Jahrhunderts wird.

2. Amerika, China, Indien, Russland, Japan denken in den Größenordnungen von Machtentfaltung und Verteidigung, mindestens Erhaltung und Erweiterung des Einflussbereichs. Viele andere Staaten wollen knapper werdende Ressourcen behalten, gewinnen oder ihre geographische Lage wenigstens gewinnbringend nutzen. Außerdem ist ein Stopp der Rüstungen zwischen Pakistan, Indien, China bis Japan und anderen Staaten nicht abzusehen. Die Programme sind souverän beschlossen, begründbar und wesentlich nur durch die jeweiligen finanziellen Fähigkeiten begrenzt. Asien hat Europa abgelöst als Region mit der größten Ansammlung von Waffen und Streitkräften. Europäische Erfahrungen der Rüstungskontrolle wären nur bedingt anwendbar, sind aber nicht gefragt.

Zwischen dem Ostrand des Mittelmeers und dem Westrand des Pazifischen Ozeans ist ein brisanter Krisenbogen entstanden, in dem sich unterschiedliche regionale, nationale, geopolitische, religiöse und Rohstoff-Interessen verknüpfen. Machtverschiebungen sind im Gange, die sich der Berechenbarkeit entziehen, z.B. im Verhältnis der drei Giganten Amerika, China und Indien. Werden Indien und China zu einer grundsätzlich beschlossenen Zusammenarbeit finden, die Amerika als Stabilitätsfaktor in Asien reduziert? Wird das Verhältnis zwischen China und Amerika kooperativer oder konfrontativer werden? Wird Indien China in zehn bis 15 Jahren überholen, was Bevölkerungszahl und technologische Modernität angeht? Die Lage in diesem Spannungsbogen wird in voraus- 
sehbarer Zeit nicht stabil werden. Kriege, sogar atomare, sind nicht auszuschließen. Europa hat dort kein entscheidendes Gewicht, auch wenn es durch Beteiligung in Konflikte hineingezogen werden kann.

3. Amerika hat die Dividende seines Sieges nach dem Ende des Warschauer Paktes ohne Bedrohung in ein gigantisches Aufrüstungsprogramm umgesetzt und nach dem 11. September 2001 praktisch ohne parlamentarische Debatte in Kraft gesetzt. Es soll seine militärische Überlegenheit zu Lande, auf dem Wasser, in der Luft und im Weltraum ausbauen und jeden anderen Staat oder Gruppierungen entmutigen, einen Wettlauf zu wagen. Das Ziel ist nicht Weltherrschaft, sondern Weltvorherrschaft, im Wesentlichen gegründet auf militärische Uneinholbarkeit. Dieses Ziel ist erreicht. Seine Überlegenheit wächst mit jedem weiteren Monat und Jahr. Diese Überlegenheit kann mindestens für die nächsten 20 Jahre vorausgesetzt werden.

Die Stärkung Amerikas werden auch alle Präsidenten, die nach dem gegenwärtigen ins Weiße Haus ziehen, verfolgen. Der mögliche qualitative Unterschied könnte darin bestehen, dass sie die Sicherheitsdoktrin abschaffen, einmotten oder abmildern, durch die der jetzige Amtsträger das Recht proklamiert hat, Krieg gegen jeden zu beginnen, den er als Bedrohung des eigenen Landes ansieht, auch präventiv, auch ohne die NATO, auch ohne Mandat der Vereinten Nationen. Es würde einen stabilisierenden Faktor einführen, wenn die USA in das Gefüge des Gewaltmonopols der UN zurückkehren würden. Als Primus inter Pares, gemäß der Definition des älteren Präsidenten George Bush, wonach Amerika das einzige Land ist, das stark genug ist, die Welt in eine neue Weltordnung zu führen. Was bedeuten würde, dass es die Regeln dieser Ordnung befolgt. Es ist nicht voraussagbar, aber zu wünschen und möglich, dass der Präsident (oder eine Präsidentin), der ab 2009 die Verantwortung haben wird, das Vertrauen in die völkerrechtliche Verlässlichkeit Amerikas wiederherstellt und das Land sich nicht von eingegangenen Verträgen befreit, die seine Rüstung behindern und neue Bindungen nur eingeht, die unbegrenzte Eigenwilligkeiten seiner Entscheidungen nicht einengen. Die Welt wird auch in den nächsten 20 Jahren mit der Existenz einer einzigen Supermacht leben, aber es wäre eine gewaltige Entspannung und Erleichterung für die Familie der Völker, wenn die amerikanische Politik wieder berechenbar würde.

Keine Änderung ist zu erwarten für das traditionelle und gewachsene Macht- und Sendungsbewusstsein Amerikas und die strategische Zielrichtung seiner Politik, im Nahen und Mittleren Osten eine Ordnung zu schaffen, die seiner machtpolitischen wie der Sicherung seiner Rohstoffinteressen entspricht.

4. Terrorismus ist eine Methode des Kampfes Schwächerer gegen Stärkere. Das unvergessliche Beispiel entstaatlichter Gewalt war der 11. September 2001. Diese Kriegserklärung Bin Ladens hat Amerika mit der globalen Kriegserklärung beantwortet. Beide Seiten führen den Kampf mit asymmetrischen Mitteln. Ihre Kriegsziele sind nicht verhandelbar. »Das Böse aus der Welt zu schaffen «, gleichbedeutend mit der westlichen Lebensart, greift weiter als die Befreiung der arabischen Welt von fremder Herrschaft. Entstaatlichte Gewalt bedroht die ganze Welt, auch Länder, die sich nicht im Krieg fühlen. Die Kriegsziele gestatten keine Kompromisse, sondern nur Sieg oder Niederlage. Dieser Krieg kann die nächsten 20 Jahre überdauern.

Neben den Netzwerken der Staaten, die den Bewegungsspielraum für die asymmetrischen Kämpfer eingeengt haben, sind die Überlegungen, auf welchem Humus die Bedrohung wächst, insgesamt zu der Antwort gekommen, dass man Menschen in zurückgebliebenen Gesellschaften begründete Aussicht auf eine bessere Zukunft schaffen muss. Diese richtige Erkenntnis verlangt Zeit und den Einsatz finanzieller Mittel, die bisher kaum erkennbar flüssig gemacht worden sind. Daneben verlangt das religiöse Element besondere Beachtung. Die fundamentale Kraft mit der Bereitschaft zur Selbsttötung wächst auch aus diesem Element. Hier ist für den Westen insgesamt ein Problem entstanden, solange er den Verdacht zulässt, er sei gegen die islamische Welt und rechtfertige also den Einsatz seiner militärischen Überlegenheit, seine imperialistische Politik, mit der Ausbreitung von Demokratie. Amerika hat seine Glaubwürdigkeit wahrscheinlich für geraume Zeit verloren und wird sie nicht zurückgewinnen, solange es nicht überzeugend nachweist, dass seine Ziele nicht zwangsläufig eine anti-islamische Politik verlangen.

Der Gefahr einer Glaubensfront zwischen Christentum und Islam kann wahrscheinlich auch begegnet werden, wenn Europa, zweifelsfrei Teil des Westens, selbstbestimmt seine Tradition des Gewaltverzichts wiederbelebt. Gewaltverzicht hat nie Verteidigungsbereitschaft in Frage gestellt, aber wohl mit ihren Grundsätzen friedlicher Koexistenz offensive Bedrohung ausgeschlossen. In diesem Krieg, dessen Ende nicht abzusehen ist, sollte jedenfalls die Gefahr eines fundamentalen Glaubenskriegs verringert, möglichst ausgeschlossen werden.

Ein Mittel dazu wäre, dass Europa anders als Amerika präventive Diplomatie und die Tradition seiner Entspannung durch Verträge und Abkommen einsetzt, um einen Kulturkampf zu verhindern. Es ist die Stärke seiner militärischen Schwäche, nicht drohen zu können. Das kann die Kräfte in der arabischen Welt ermutigen, die in konstruktiver Zusammenarbeit mit der westlichen Welt mehr Zukunftsaussichten sehen, als in militanter Abwehr.

Nach den vier wesentlichen Elementen des globalen nun zum europäischen Befund. Der Begriff Europa steht für eine Idee und die erfolgreichste Neugründung einer internationalen Organisation nach dem Zweiten Weltkrieg. Die Idee heißt Selbstbestimmung Europas mit dem Ziel der globalen Handlungsfähigkeit. Die Organisation heißt Europäische Union und stellt den bedeutendsten freiwilligen Zusammenschluss mit der Qualität dar, dass ihre Staaten nicht mehr kriegführungsfähig gegeneinander sind, die Vorteile eines riesigen gemeinsamen Marktes so groß sind, dass immer noch mehr Länder hinein wollen und ihre Organe wirtschaftspolitische Kompetenzen von den Nationalstaaten übertragen bekommen haben, die sie insoweit international handlungsfähig gemacht haben.

Das Ende des Ost-West-Konflikts bedeutete auch für die EU den elementarsten Einschnitt ihrer Geschichte. Die Selbstbestimmung Europas, vorher nur wirtschaftlich gemeint, weil für die Sicherheit Amerikas Garantie unentbehrlich war, wur- 
de nun auch verteidigungspolitisch möglich: jedenfalls konventionell, weil die Bedrohung aus dem Osten weg war und der bis heute bleibende amerikanische atomare Schirm daran nicht hindert.

Die historisch richtige Erweiterung der Gemeinschaft verschob ihr Gleichgewicht nach Osten. Wichtige Grundsatzentscheidungen der EU verlangen die Zustimmung osteuropäischer Staaten, faktisch eine Garantie, dass auch die Selbstbestimmung Europas nicht anti-amerikanisch sein kann. Die Vertiefung, sprich Regierbarkeit der 27 Mitglieder ist vorläufig durch die Blockierung des sogenannten Verfassungsvertrages durch Frankreich und die Niederlande gescheitert. Eine Entscheidung über die Grenzen der EU, also ihre Erweiterung, ist offen. Eine Entscheidung über den Beitritt der Türkei wird kaum vor 2016 fällig werden.

Vorher jedenfalls wird sich das Problem der Geschlossenheit für die weitere innere Entwicklung stellen. Das Erfolgsprinzip der Gemeinschaft beruht auf der Übertragung von nationaler Souveränität auf die Gemeinschaft mit der Begrenzung, dass ihre Länder keine Föderation anstreben, sondern im Nebeneinander der Nationalstaaten die Zukunft gestalten wollen. Ein Bruch der Geschlossenheit ist erfolgt, als nicht alle Mitglieder dem Schengener Abkommen beigetreten sind, und ein weiterer sehr viel bedeutenderer, als nicht alle Mitglieder den Euro einführen wollten oder konnten. Die Entscheidung über die eigene Währung gehört zu den vornehmsten Rechten jeder Regierung. Der Verzicht darauf zu Gunsten einer gemeinsamen Währung bedeutete einen Qualitätssprung in die Vollintegration einer übernationalen Einheit. Der Euro ist ein globaler Faktor neben den Währungen Amerikas, Chinas und Japans geworden, Ausdruck europäischer Selbstbestimmung nicht gegen, sondern neben dem Dollar.

Gleiches würde gelten für die beiden offenen Felder der Verteidigung und der Außenpolitik. Qualitative Schritte auf diesen Gebieten, die nicht alle Länder mitgehen, waren bereits in der gescheiterten Verfassung von allen beschlossen und vorgesehen. Sie liegen bei der Verteidigung näher und könnten den Weg erleichtern für das seit 40 Jahren proklamierte Ziel, dass Europa mit einer Stimme spricht. In der Konsequenz muss das zu dem Versuch führen, die Länder, die dazu fähig und willens sind, zu einem engeren Zusammenschluss zu führen, der für jeden weiteren Staat offen ist. Der Zusammenschluss zu einem solchen Kern würde die Entwicklung zu einer europäischen Armee einleiten.

Sie würde in einem Punkt keine Veränderung zur gegenwärtigen Situation bedeuten: Unter den globalen Schwergewichten wäre es offensichtlich aussichtslos, militärisch konkurrieren zu wollen. Von den Finanzen einmal abgesehen, ergäbe es auch keinen Sinn. Warum sollte Europa Fähigkeiten anstreben, die Amerika schon im Übermaß hat? Gegen welchen Feind? Amerika ist ein Verbündeter, gegen den wir uns nicht schützen müssen. China, Indien und Russland bedrohen uns nicht. Amerikas globale Ziele als Hilfstruppe zu unterstützen, ist nicht attraktiv. Der Unterschied zur Gegenwart wäre die Geschlossenheit der Verfügung, die in der Struktur der Einheitlichkeit des Euro ähnlich über den Einsatz dieses europäischen Instruments entscheidet.
Aber auch in der Pause bis zu einer europäischen Armee müssen die Fähigkeiten der europäischen Staaten ausreichend und militärisch modern entwickelt werden, um seine politischen Interessen wirksam und überzeugend vertreten zu können. Das heißt global Frieden und Stabilität zu fördern, zu sichern oder wiederherstellen zu helfen. Und im Übrigen, um eingegangene Verpflichtungen nicht zuletzt auf dem eigenen Kontinent garantieren zu können. Solange die Gefahr droht, dass sich die Beteiligten im ehemaligen Jugoslawien wieder an die Kehle gehen, bleiben europäische Streitkräfte dort unentbehrlich, die amerikanische Truppen dort nicht mehr brauchen.

Selbst in Afghanistan ist zu beobachten, dass Friedenserhaltung und Friedenserzwingung durchaus unterschiedliche Fähigkeiten verlangen. Daraus resultieren unterschiedliche Ausrüstungen. Die amerikanischen Fähigkeiten, einen Irak-Krieg zu führen, braucht Europa nicht. Im Ansatz sind selbstständige Führung und Einsatzfähigkeiten schon vorhanden. Eine sicherheitspolitische Debatte über unterschiedliche, vorwiegend globale amerikanische und die vorwiegend europäischen Verantwortungen ist überfällig. Sie würde der Klarheit von Arbeitsteilung und der Europäisierung Europas dienen.

In den nächsten Jahren wird in diesem Zusammenhang die Frage unabwendbar werden, ob England dieses Ziel überhaupt noch teilt. Seit es die europäische Bewegung gibt, haben alle englischen Regierungen mit bewundernswerter Konsequenz und unbestreitbarem Erfolg den Vorzug traditioneller Sonderbeziehungen zu Amerika gewahrt und eine unrevidierbare Bindung an den Kontinent abgelehnt. Unabhängig, welche Partei den Premierminister stellte, haben alle die Politik verfolgt: Zusammenarbeit ja, aber keine Integration, die den Vorrang des Verhältnisses zu Amerika auch nur schmälern könnte; immer drängend auf die Erweiterung der Gemeinschaft, die sie immer schwerer regierbar macht, aber bremsend bei ihrer Vertiefung; logisch keinen Beitritt zum Euro, der praktisch unwiderrufbar wäre, aber werbend für die Vorteile eines riesigen gemeinsamen Marktes.

England hat sich eine einzigartige Sonderstellung in und gegenüber Europa verschafft: Praktisch verhindert es die europäische Selbstbestimmung, weil damit sein Sonderverhältnis zu Amerika beendet würde. Die Selbstbestimmung Europas verlangt eine Emanzipation von Amerika und genau die Emanzipation von Amerika macht England keinesfalls mit. Diese Haltung entspricht seinen Interessen. Sie steht im Widerspruch zu den Interessen eines selbstbestimmten Europas, jedenfalls zu seinem beschlossenen Ziel. Es ist kein Element zu erkennen, das England zu einer grundsätzlichen Neuorientierung veranlassen könnte. Ein sensationelles Angebot, unter welchen Bedingungen es sich dem Ziel der politischen Selbstbestimmung unwiderruflich anschließen würde, ist auch in den nächsten 20 Jahren nicht zu erwarten. Wenn Europa sich nicht praktisch dem englischen Interesse unterordnen will, muss es den eigenen Weg der Länder gehen, die dazu willens und fähig sind, notfalls auch ohne England wie beim Euro. Wenn die Chancen zu einem Global Player nicht genutzt werden, würde Europa sich weltpolitisch marginalisieren und manipulierbar machen. 
Jeder Überblick über die unterschiedlichen globalen, regionalen und europäischen Entwicklungen zeigt ihre Verflochtenheit. Offensichtlich ist der nationale Hebel zu schwach, um deutsche Sicherheitsinteressen im Alleingang wirksam durchsetzen zu können. Sie sind auf diesem Sektor am besten im europäischen Verbund zu fördern.

Auch dafür sollten wir uns zwei Faktoren bewusst machen, die Haltung und Methodik bestimmen. Der eine ist nationale Identität, der andere heißt Macht.

Das deutsche Selbstverständnis ist in den Jahrzehnten der Teilung ziemlich verkümmert. Gemäß den Realitäten konnte man einerseits die NATO unter Führung Amerikas, andererseits das sozialistische Lager unter Führung der Sowjetunion eine Staatsraison nennen. Der Nation konnte dennoch keine Seite entkommen. Die Versuche dazu waren antagonistisch da und dort, die Last der jüngsten Geschichte zu erleichtern. Seit der Einheit ist die Tradition der alten Bundesrepublik fortgesetzt worden, die Hoffnung und ehrliche Absicht zugleich war, die Geschichte der Nation hinter uns zu lassen und eine europäische Föderation, am besten die Vereinigten Staaten von Europa zu schaffen.

Aber keiner unserer westlichen Partner wollte seiner Nation entkommen. Und für die neuen Partner im Osten war die Nation Quelle und Ausdruck ihrer Souveränität und Freiheit. Einen immer noch denkbaren Weg hat Joschka Fischer in einer großartigen Rede vor der Humboldt-Universität entwickelt. Auch das erwies sich als Traum in dem Augenblick der Verhandlungen über eine europäische Verfassung: Keiner der 24 Partner wollte den Traum teilen. Sie wollten die Zukunft im Nebeneinander der Nationalstaaten gestalten. Deutschland, der 25. Staat schloss sich an. Die Voraussage ist kaum ein Risiko, dass in den nächsten 20 Jahren das Projekt einer Föderation nicht wieder auf den Tisch der Regierungen kommen wird.

Deutschland muss also wie jeder andere europäische Staat seine Interessen souverän formulieren und vertreten. Völkerrechtlich ist das kein Problem, wohl aber psychologisch; denn kein anderes Volk teilt unsere Vergangenheit. Von fast allen Partnern in Europa, sogar in Israel, werden Tiefe und Ernsthaftigkeit der deutschen Auseinandersetzung mit seiner Vergangenheit anerkannt bis zu dem Punkt, dass wir ziemlich isoliert den Einsatz deutscher Soldaten inzwischen nur noch im Zusammenhang mit Israel diskutieren.

Auschwitz, das erstmalige und hoffentlich einmalige Verbrechen der industriellen Menschenvernichtung, wird auch in 500 Jahren unvergessen sein. Aber selbst diese Ungeheuerlichkeit darf nicht zu einem demütigenden Joch werden, das uns immer vor wichtigen Entscheidungen in Gegenwart und für die Zukunft fragen lässt, ob wir das wegen Auschwitz dürfen, als ob den Verbrechern von damals eine Art indirektes Mitspracherecht einzuräumen wäre. Die deutsche Vergangenheit darf die europäische Zukunft nicht belasten.

Europa verlangt nicht die Aufgabe des Nationalstolzes. Im Gegenteil: $\mathrm{Zu}$ den Bausteinen gehört, dass dazu ein guter Spanier, ein guter Ungar, ein guter Tscheche und ein guter Niederländer verlangt ist. Man kann zweifeln, ob ein schlech- ter Deutscher ein guter Europäer sein kann. Wir können von unseren Nachbarn lernen: Nationalstolz ist kein Nationalismus. Es gibt sehr genau erkennbar einen französischen, einen polnischen, einen italienischen, einen türkischen Weg. Das deutsche Selbstverständnis gestattet nicht nur, es verlangt einen deutschen Weg, vor dem weder wir selbst noch andere Angst zu haben brauchen. Das Heranwachsen eines Europas der Nationalstaaten verlangt in seiner Mitte ein Deutschland mit einem gesunden Patriotismus.

»Es wird die Aufgabe der deutschen Politik sein dafür zu sorgen, dass das Nationalbewusstsein des deutschen Volkes ein Gleichgewicht findet. Das nach Exzess und Negation das wahre Maß des Nationalgefühls gefunden wird, das frei ist von Überheblichkeit und getragen ist von Mut und gutem Willen gegenüber allen Menschen und allen Nationen. Ob das gelingt, wird die eigentliche Entscheidung über das Schicksal der Demokratie in Deutschland sein. « Aufregend, dass Brandt vor 40 Jahren an der Harvard-Universität die Aufgabe der deutschen Politik von heute formuliert hat.

Die Vokabel Macht ist nicht beliebt und wird im Zusammenhang mit Außenpolitik in Deutschland ungern benutzt. Dennoch spielen Machterhaltung, Machterweiterung, Machtwiederherstellung international eine große Rolle, ob wir wollen oder nicht. Die Abwendung von der Macht lässt sie nicht verschwinden. Wenn wir sie nicht nutzen, wird sie gegen uns genutzt. Die viel gescholtene Realpolitik ist eine Realität. Mit anderen Worten: Deutschland muss wieder machtgewohnt werden. Machtgewöhnung ist ein anderes Wort für Normalität, die Normalität der Staaten.

Richard von Weizsäcker verdanken wir die blendende Formulierung, dass die Deutschen zwischen der Machtbesessenheit des Dritten Reiches und der Machtvergessenheit der alten Bundesrepublik gependelt haben. Das bedeutet, dass wir vor der Aufgabe stehen, das Gleichgewicht zu finden, das Gleichgewicht zwischen Machtverzicht und Überheblichkeit. Das ist Normalität. Das bedeutet für einen unentbehrlichen Staat in der Mitte Europas die unentbehrliche Verantwortung, Macht nicht gegen andere und über andere, also keine Vorherrschaft, sondern für etwas und mit anderen entfalten zu wollen. In der Verfassung wird das als Gebot formuliert, gleichberechtigt »in einem vereinten Europa dem Frieden der Welt zu dienen«. Insoweit wird angemessener Gebrauch von Macht zur Pflicht.

Für die deutschen Sicherheitsinteressen gilt unverändert die Orientierung Willy Brandts:

»Friede ist nicht alles, aber ohne Frieden ist alles nichts. «In der Tat: Es gibt andere Werte und sogar Grundwerte, für die wir uns einsetzen, aber unbestreitbar können Menschenrechte, Menschenwürde, Demokratie, Umweltbewahrung, Kampf gegen Hunger, Alphabetisierung und politische Selbstbestimmung in einem Krieg weder geschützt noch gefördert werden. Die Erhaltung des Friedens als oberster Wert wird nur in einer Lage relativiert, in der nach einem anderen Wort von Brandt: »Im Zweifel für die Freiheit « zu entscheiden ist. In eine solche existenzielle Bedrohung nicht zu kommen, ist das Ziel deutscher Politik. 
Unsere Sicherheitsinteressen weisen den Weg zu diesem Ziel. Auf der Grundlage unserer Geschichte und gestützt auf die eingegangenen Verträge wollen wir die deutsche Souveränität einsetzen, um die Europäische Union, die den Krieg zwischen ihren Mitgliedern überwunden hat, zu befähigen, ihre Selbstbestimmung zu vollenden und global für Stabilität und Frieden zu wirken.

Diese Grunddefinition unserer Interessen geht von der Verantwortung aus, die das souveräne, vereinte Deutschland für alle seine Handlungen oder Unterlassungen erhalten hat. Der rechtliche Rahmen ergibt sich aus der Tatsache, dass wir keiner internationalen Organisation angehören, die vitale Fragen mit Mehrheit entscheidet. Das gilt für die UN, die NATO und für die nächste Zukunft (leider) noch für die EU.

Die mehrfach benutzte Formulierung: »Wir können uns den Wünschen der internationalen Gemeinschaften nicht entziehen «, ist nicht nur falsch, sondern letztlich verfassungswidrig. Falsch, weil der Mangel an Automatik nicht nur für alle anderen Partner dieser Organisationen gilt, sondern auch für Deutschland. Verfassungswidrig ist eine Formulierung, die suggeriert, dass Regierung und Parlament letztlich nicht mehr die festgelegte Entscheidungsfreiheit ausüben können, und die darüber hinaus den Artikel 26 der Verfassung nicht berücksichtigt. Dieser Artikel verbietet die Teilnahme an einem Angriffskrieg, also an einem Krieg ohne Mandat der UN. Er ist in dieser Rigorosität einzigartig. Er singularisiert Deutschland, auch in Fällen, in denen sogar eine Mehrheit von Verbündeten sich als willig erklärt, Krieg auch ohne Mandat der UN zu führen. Es ist durchaus kein Nachteil, wenn die Völkergemeinschaft weiß, dass Deutschland sich keinesfalls an völkerrechtlich zweifelhafter Gewaltanwendung beteiligen wird.

Politisch ist die Abwägung unserer Interessen durch den Rahmen bestimmt, dass wir auf der einen Seite für Vorschläge und Ideen, wie gut sie auch sein mögen, genügend unterstützende Partner brauchen, um sie durchzusetzen; auf der anderen Seite reichen Gewicht und Lage des Landes bei Fragen europäischer Dimension, eine Entscheidung gegen vitale deutsche Interessen zu verhindern. Dieser Spielraum deutscher Außen- und Sicherheitspolitik ist fast ideal zu nennen: Er garantiert für unsere Partner Berechenbarkeit und behindert weder Anregungen noch eigene Initiativen.

Rüstungskontrolle und Abrüstung waren einmal ein Markenzeichen deutscher Politik und der SPD im Besonderen. Es lohnt hier nicht, die Gründe nachzuzeichnen, warum das verloren gegangen ist. Die über alle Erwartungen großen Erfolge haben sich in dem bedeutendsten konventionellen Rüstungsabkommen der Geschichte niedergeschlagen, das sich sogar nach dem Ende der europäischen Spaltung bewährt hat, und in der Charta von Paris, die 1990 das Grundgesetz europäischer Stabilität und Sicherheit formuliert hat. Es heißt Gewaltverzicht für alle Änderungen international anerkannter Grenzen, wobei Änderungen friedlich vereinbart werden können. Das Prinzip ermöglichte die deutsche Einheit wie die Teilung der CSSR. Der Befund unserer Welt verlangt die Wiedergeburt von Rüstungsbegrenzung und Rüstungskontrolle. Das ist eine Aufgabe, bei der Europa Erfahrungen und Interessen hat. Die positiven Erfahrungen liegen auf der Hand. Die Interessen ergeben sich aus seiner Tradition, seinen Werten und seinem Willen zur Selbstbestimmung. Eine europäische Initiative zur Renaissance von Rüstungskontrolle und Rüstungsbegrenzung könnte eine weltweite Wirkung entfalten. Sie könnte während der deutschen EU-Präsidentschaft vorbereitet werden.

Die Risiken ungebändigter Aufrüstung wachsen, wenn die Welt nicht versteht, dass sie Regeln für eine globale Zwangspartnerschaft erarbeiten muss, vergleichbar der Zwangspartnerschaft der gemeinsamen Sicherheit während des Ost-West-Konflikts. Der Rüstungswettlauf bei neuen Raketen-, Atom- und Weltraumwaffen würde einen Weltgipfel der Vereinten Nationen zu Rüstungsreduktion, Nichtverbreitung und Massenvernichtungswaffen rechtfertigen. Deutsche Anregungen dazu sollten entwickelt werden.

Außerdem gibt es einen Klärungsbedarf. Die Sicherheitsdoktrin Amerikas ist durch den Entwurf einer neuen Nukleardoktrin ergänzt worden. Nach ihr können »Mini-Nukes« eingesetzt werden, wenn die politische und militärische Führung das zum Erreichen der gesetzten Ziele in einem Konflikt für richtig hält. Das bedeutet nicht nur die Senkung der atomaren Schwelle, sondern die Nutzung in einer konventionellen Auseinandersetzung, auch im Krieg gegen den Terror. Der NATORat dürfte dabei kein größeres Mitspracherecht erhalten als zur Zeit des Ost-West-Konflikts, also praktisch keins. Eine schnelle Eingreiftruppe zur Unterstützung einer amerikanischen Aktion könnte sich also unversehens in einem atomaren Szenario finden, zumal die Stabschefs korrekt darauf hingewiesen haben, dass es keinen Vertrag gibt, der den Einsatz atomarer Waffen verbietet. Eine derartige neue Doktrin legt eine förmliche Vereinbarung nahe, keine Mini-Nukes einzusetzen, wo europäische Krisenreaktionskräfte eingesetzt werden sollen.

Die deutsche Definition der strategischen Partnerschaft mit Russland ist in der Erklärung der Großen Koalition bekräftigt worden. Sie entspricht dem Interesse, auch ohne die Perspektive einer russischen Mitgliedschaft in der EU, dieses große Land in eine verlässliche Nähe zu Europa zu bringen. Das liegt politisch, aber eben auch sicherheitspolitisch in der Dimension des »Europäischen Hauses«. Verlässliche Stabilität für Gesamteuropa ist erstrebenswert. In diesen Zusammenhang würde sich ein deutscher Vorschlag einfügen, die gegenseitige Drohung von NATO und Russland zum Erstgebrauch von Atomwaffen abzuschaffen; sie passt nicht mehr in die Landschaft beiderseitiger strategischer Partnerschaft und wachsender Freundschaft. Deutschland kann dafür sorgen, dass Rüstungskontrolle und Abrüstung wieder eine Zukunft erhalten, die mit dem deutschen Namen verbunden wird. 